\title{
An Overview of Localization for Wireless Sensor Networks
}

\author{
${ }^{1}$ Vadivukkarasi. K and ${ }^{2}$ R. Kumar \\ ${ }^{1,2}$ Department of Electronics and Communication, SRM University
}

\begin{abstract}
Localization is an important issue for location-dependent applications of wireless sensor networks. In Sensor networks, data collected by sensor nodes need to be tagged with time and location information. Localization techniques are used to determine the location information of a sensor node. It enables efficient routing, provides power saving, assists in the applications like target tracking, useful in locating the source of the data. The localization can be categorized as range-free or range-based schemes based on whether the range information is used among the localization process. Because of the hardware limitations of the network devices, solutions in range-free localization are being pursued as a cost-effective alternative to more expensive rangebased approaches. In this paper described recent techniques used for localization.
\end{abstract}

Keywords: Wireless sensor networks, localization, anchor, multi hop, range free and range based.

\subsection{Wireless Sensor Networks (WSN)}

\section{Introduction}

A sensor network is composed of a large number of sensor nodes that are densely deployed inside or very close to the phenomenon. A WSN is generally composed of a centralized station (sink) and tens, hundreds, or perhaps thousands of tiny sensor nodes. With the integration of information sensing, computation and wireless communication, these devices can sense physical phenomenon, process the raw information, and share the processed information with their neighboring nodes[1]. WSN architecture shown in figure.1. It consists of sensor field, sensor nodes, sink, communication medium and user. Some of the characteristic features of sensor networks include the following:

- Sensor nodes are densely deployed

- Sensor nodes are prone to failures

- The topology of a sensor network changes very frequently

- Sensor nodes are limited in power, computational capacities and memory.

- Sensor nodes may not have global identification because of the large amount of overhead and large number of sensors.

The hardware components of a sensing node include a sensing and actuation unit, a processing unit, a communication unit, a power unit and other application dependent units. WSNs are special type of distributed network system that is similar to data base, real-time and embedded systems. Each sensor node needs a operating system (OS) that can control the hardware. WSN need a new type of operating system, considering their special characteristics such as small size, real time support, efficient resource management mechanisms, reliable and efficient code distribution, power management and generic programming interface up to sensor application software. The platform used for WSNs are TinyOS, Mate, MagnetOS, MANTIS, OSPM and EYES OS[22].

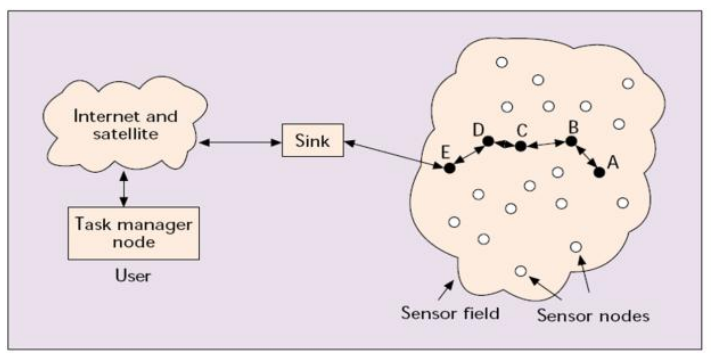

Fig. 1. Wireless sensor network Architecture

The sensor nodes available in the markets are generic nodes and gateway node. Generic nodes are used to measure task measurements such as light, temperature, humidity, barometric pressure, velocity, acceleration, acoustics and magnetic field. Gateway nodes (sink) are used to collect data from generic sensors and relay them to the base station. 


\subsection{WSN design factors:}

a) Fault Tolerance: Fault tolerance is the ability to sustain sensor network functionalities without any interruption due to sensor node failures.

b) Scalability: Scalability measures the density of the sensor nodes.

c) Production Costs: The cost of a single node must be low given the amount of functionalities

d) Hardware Constraints: All these units combined together must provide extremely low power and extremely small volume

e) Sensor Network Topology: Deploying and managing a high number of nodes in a relatively bounded environment requires special techniques.

f) Environment: Sensor networks often are expected to operate in an unattended fashion in dispersed and/or remote geographic locations: Nodes may be deployed in harsh, hostile, or widely scattered environments. Such environments give rise to challenging management mechanisms.

g) Transmission Media: Sensor networks often operate in a bandwidth and performance constrained multihop wireless communication medium. These wireless communications links operate in the radio, infrared, or optical range.

h) Power Consumption: The sensor node lifetime typically exhibits a strong dependency on battery life. Power consumption can therefore be allocated to three functional domains: sensing, communication, and data processing, each of which requires optimization.

\subsection{Protocol Stack for WSN}

The protocol stack for WSN is shown in figure.2. Upper layers: In-network applications, including application processing, data aggregation, external querying query processing, and external database Transport Layer: Transport, including data dissemination and accumulation, caching, and storage. Network Layer: Networking, adaptive topology management and topological routing. Data Link layer (contention): channel sharing (MAC), timing, and locality. Physical medium: communication channel, sensing, actuation, and signal processing.

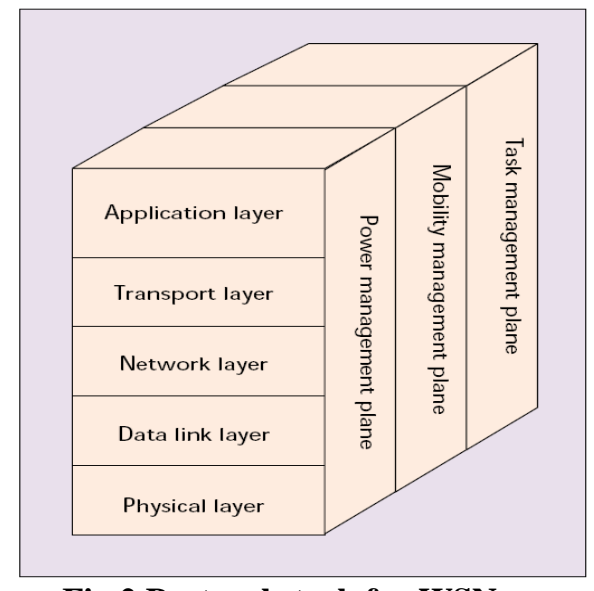

Fig.2.Protocol stack for WSN

\subsection{Network Services for WSN}

a) Synchronization: Time synchronization is important for routing, power conservation, Lifetime, Cooperation, Scheduling of WSN. It minimizing uncertainty errors over long periods of time and dealing with precision

b) Coverage: It is important in evaluating effectiveness. Degree of coverage is application dependent. It optimizes for better energy conservation

c) Compression and aggregation: Data compressed before transmitting to base and decompression occurs at the base station. Data is collected from sensor nodes and combined together to transmit to base station. It's used in cluster based architecture.

d) Security: protocols have to monitor, detect, and respond to attacks .It has done for network and data-link layer (can be improved) should be done for different layers of the protocol stack.

e) Localization: It is used to determine node's location.

The paper is organized as follows: Need of localization is given in the next section. Basic localization techniques presented in the section 3. Section 4 gives improved algorithms based on range based approach. Section 5 gives improved algorithms based on range free approach. The last section is to conclude this paper. 


\section{Localization}

Localization is the process by which sensor nodes determine their location. In simple terms, localization is a mechanism for discovering spatial relationships between objects. It is important when there is an uncertainty of the exact location of some fixed or mobile devices. An effective localization algorithm can then use all the available information from the wireless sensor nodes to infer the position of the individual nodes[12].

\subsection{Need of localization}

Having knowledge of sensor locations allows one to use diverse sensor data more efficiency, plan resource routing priorities to support network services or perform surveillance effectively. Many applications, such as object tracking, location based routing, coverage management and collaborative signal processing, require that sensor nodes be able to automatically and accurately determine their absolute or relative (with respect to other nodes) locations. Consider the example where a sensor network is used to detect a fire event in a forest. Once a sensor node has detected that the temperature is higher than a certain threshold, it sends a message to the central authority by relaying through other nodes in a multi-hop manner. The message needs to indicate the location of the node which detected the event[2]. Thus, localization of sensor nodes is important in some applications.

Anchor (or) beacon nodes and unknown nodes are the two types of nodes employed in localization. A beacon is a node aware of its location (e.g. equipped with GPS)[3]. The nodes of initially unknown positions will be called unknown nodes. After the sensor node has been deployed, the mobile beacon assists the unknown nodes in localizing themselves. The mobile beacon can be a human operator, an unmanned vehicle deployed with the sensor network, or in the case of a deployment from a plane, the plane itself. With regard to the mechanisms used for estimating location, the localization protocols are divided into two categories: range-free and range-based. In the range free approaches, the algorithms do not need range hardware support and are immune to range measurement errors while providing less accurate but still acceptable localization results. In the range-based approaches, the algorithms require more sophisticated range hardware support to acquire absolute point-to-point distance estimates or angle estimates for calculating locations. The range-based approaches provide more accurate localization results than the range-free algorithms. Because of the hardware limitations of WSN devices, the range-free localizations are being pursued as a cost-effective alternative to more expensive range-based approaches.

\section{Basic localization techniques for Range based and Range free localization scheme}

Node localization has been the topic of active research and a number of systems have been proposed over the past few years. Many of those systems fall into one of three classes or a combination of them. The first class includes range free algorithms, which assume that there is no distance/angle information available at each node. Hence, they try to use the basic proximity information available at each node, i.e. which nodes are nearby. For example, the GPS-less system estimates the position of a node as the centroid of positions of connected anchor nodes. In general, range-free techniques provide the lowest level of accuracy among the three classes. The second class employs a number of specialized, anchors, nodes that know their positions usually using GPS[4]. The rest of the nodes try to estimate their positions relative to these anchors. For example, in the iterative multilateration method, an unknown node that is connected to at least three anchors estimates its position by solving a system of equations. Once a node estimates its position it becomes an anchor and assists other unknown nodes in estimating their positions by propagating its own location estimate through the network. Most of the methods in this class require a high percentage of anchor nodes.

The third class of localization systems tries to compute nodes positions without the use of anchor nodes. In this case, instead of computing absolute node positions, the algorithm estimates nodes positions relative to a coordinate system established by a reference group of nodes. Relative positioning can be sufficient for many applications to work efficiently, for example, location-aided routing. Moreover, a relative coordinate system can still be transformed to absolute coordinate system by using only three anchor nodes in case of 2-D (or four anchors in case of 3-D). Schemes in this class can be range-free or range-based.

\subsection{Range Based schemes}

Range-based schemes rely on the range measurements (received signal strength (RSS), time of arrival (TOA), time difference of arrival (TDOA) and angle of arrival (AOA)) among the nodes.

\subsubsection{Received Signal Strength Indication (RSSI):}

The RSSI model is based on the fact that signal strength diminishes with distance. Thus the distance between the source and the receiver could be found out by the strength of the radio signal received. 


$$
\mathrm{d}=\left(\mathrm{xP}_{\mathrm{t}} / \mathrm{P}_{\mathrm{r}}\right)^{1 / 2}
$$

' $\mathrm{d}$ ' is the distance between the transmitter and the receiver, ' $\mathrm{P}_{\mathrm{r}}$ ' is the received power, ' $\mathrm{P}_{\mathrm{t}}$ ' is the transmitted power. The demerits of RSSI method is that it is easily affected by multi-path fading, shadowing, scattering and also in the non-line of sight condition[12].

\subsubsection{Time Difference of Arrival method (TDoA)}

The time difference of arrival method (TDoA) was also devised to calculate the distance of the sensor nodes. This method used extra speaker and a microphone. The sender nodes sends the radio signal at first and after some time it again sends a sound 'chirp'. The receiver then detects the radio signal at time $T_{\mathrm{r}}$ and the sound at time $T_{s}$ after some time delay $T_{d}$. then uses this information to calculate the distance between the source node and the destination node.

$$
\mathrm{d}=\left(\mathrm{S}_{\mathrm{r}}-\mathrm{S}_{\mathrm{s}}\right) *\left(\mathrm{~T}_{\mathrm{s}}-\mathrm{T}_{\mathrm{r}}-\mathrm{T}_{\mathrm{d}}\right)
$$

' $\mathrm{d}$ ' is the distance between the source and the receiver, $\mathrm{S}_{\mathrm{r}}$ is the radio signal, $\mathrm{S}_{\mathrm{s}}$ is the sound signal. The disadvantage of this method was that the sensor nodes required an extra microphone and speaker to be built in. The signal speed is also affected by humidity and temperature and for some condition the line of sight.

\subsubsection{Angle of Arrival (AoA) scheme}

The Angle of Arrival (AoA) method is proposed to calculate the distance of the sensor nodes. This method used Radio and microphone arrays to calculate the distance. Here the microphones hear the transmitted signal and analyze the phase or the time difference of the arriving signals at the microphone and thus calculates the angle of the arrival signal. The demerit of this method is that the hardware used is expensive and more bulkier as compare to TDoA. Also this method is affected by the multi-path effects, shadowing, scattering and also when there is no line of sight[10].

\subsubsection{Range based indoor localization scheme}

RADAR is a range-based indoor localization system that measures RSS at all positions in the entire building and records the RSS into a database during the calibration phase. In the localization phase, the location and the orientation of a user is determined by finding the best match of a set of RSS measurements from the database. The cricket indoor localization support system utilizes a combination of RF and ultrasound measurements to provide location information to users[6][8].

\subsubsection{The Self-Positioning Algorithm (SPA)}

The Self-Positioning Algorithm (SPA) is to find relative positions in the network using distance measurements between the nodes. Each node builds its own local coordinate system, estimates the positions of one-hop neighbours using triangulation and broadcasts this information to a11 the nodes in the network to build a global network coordinate system. In addition to the large communication overhead needed to build the global network topology, the achievable accuracy is still limited. Instead of forming a local coordinate system at each node like SPA[10][11, we build a cluster-wide coordinate system only at each gateway node. In this case we gain the following benefits:

(1) since the gateway node is assumed to be less-resource constrained, we can use non-linear optimization techniques to estimate the nodes' position more accurately; (2) the communication overhead to build global network topology is reduced since only gateway nodes communicate with each other. While other anchor free mechanisms consider nodes that 1 or 2 hops away, we estimate the position of nodes that are within k-hops from the gateway node since the gateway serves the entire cluster. We refer to ' $\mathrm{k}$ ' as the cluster radius and it is a parameter in our algorithm. A problem that occur is the error accumulated in the node position as it becomes multihop away from the gateway node. Clearly, as ' $\mathrm{k}$ ' increases the number of nodes in the cluster (Cluster Size) increases and the total number of clusters in the network decreases. One of the contributions of this paper is to show how the error accumulates, as the node becomes k-hop away from the gateway node, and what factors affect this error accumulation.

\subsection{Range Free Schemes}

The range based method is defined by protocols that use absolute point-to-point distance estimates (range) or angle estimates for calculating location. The range free method makes no assumption about the availability or validity of such information. Because of the hardware limitations of WSN devices, solutions in range-free localization are being pursued as a cost-effective alternative to more expensive range-based approaches[5].

\subsubsection{Centroid algorithm}




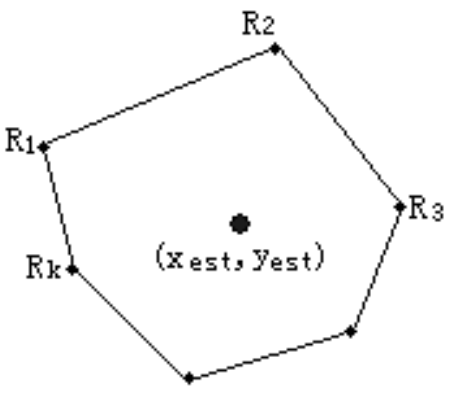

Fig.3.Centroid algorithm

The main idea of centroid algorithm is shown in figure.3. Multiple nodes in the network with overlapping regions of coverage serve as reference points (labeled $R_{1}$ to $R_{k}$ ). They are situated at known positions, $\left(\mathrm{X}_{1}, \mathrm{Y}_{1}\right)$ to $\left(\mathrm{X}_{\mathrm{k}}, \mathrm{Y}_{\mathrm{k}}\right)$, and form a regular mesh and transmit periodic beacon signals containing their respective positions with a period $\mathrm{T}$. The unknown node collects all positions that it receives from various reference points, denoted as $\mathrm{R}_{1}, \mathrm{R}_{2}, \ldots, \mathrm{R}_{\mathrm{k}}$, and localizes itself to the centroid of these reference points. The estimated coordinates $\left(\mathrm{X}_{\mathrm{est}}, \mathrm{Y}_{\mathrm{est}}\right)$ of unknown node are computed by the centroid formula:

$$
\begin{aligned}
& \mathrm{X}_{\mathrm{est}}=\left(\mathrm{X}_{1}+\mathrm{X}_{2}+\ldots \mathrm{X}_{\mathrm{k}}\right) / \mathrm{K} \\
& \mathrm{Y}_{\mathrm{est}}=\left(\mathrm{Y}_{1}+\mathrm{Y}_{2}+. . \mathrm{X}_{\mathrm{k}}\right) / \mathrm{K}
\end{aligned}
$$

The distinguished advantage of this centroid localization scheme is its simplicity and ease of implementation.

\subsubsection{DV-HOP Scheme}

DV-Hop localization uses a mechanism that one anchor broadcasts a beacon to be flooded throughout the network containing the anchors location with a hop-count parameter initialized to one. Each receiving node maintains the minimum counter value per anchor of all beacons it receives and ignores those beacons with higher hop-count values. Beacons are flooded outward with hop-count values incremented at every intermediate hop. Through this mechanism, all nodes in the network (including other anchors) get the shortest distance, in hops, to every anchor. The hop count for a single anchor A, is shown in figure.4.

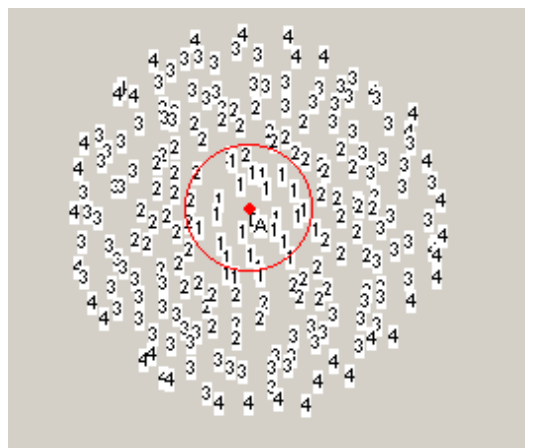

Fig.4.DV-Hop

The average single hop distance is then estimated by anchor "i" using the following formula:

$$
\text { Hopsize }_{\mathrm{i}}=\sum\left[\sqrt{ }\left(\left(\mathrm{x}_{\mathrm{i}}-\mathrm{x}_{\mathrm{j}}\right)^{2}+\left(\mathrm{y}_{\mathrm{i}}-\mathrm{y}_{\mathrm{j}}\right)^{2}\right)\right] / \sum \mathrm{h}_{\mathrm{j}}
$$

In this formula, $\left(\mathrm{x}_{\mathrm{j}}, \mathrm{y}_{\mathrm{j}}\right)$ is the location of anchor $\mathrm{j}$, and $\mathrm{h}_{\mathrm{j}}$ is the distance, in hops, from anchor $\mathrm{j}$ to anchor $\mathrm{i}$. Once calculated, anchors propagate the estimated HopSize information out to the nearby nodes[9].

Once a node can calculate the distance estimation to more than 3 anchors in the plane, it uses triangulation (multilateration) to estimate its location. 


\subsubsection{Amorphous Localization algorithm}

This algorithm used independently from DV-Hop, uses a similar algorithm for estimating position. First, like DV-Hop, each node obtains the hop distance to distributed anchors through beacon propagation. Once anchor estimates are collected, the hop distance estimation is obtained through local averaging. Each node collects neighboring nodes hop distance estimates and computes an average of all its neighbor values. Half of the radio range is then deducted from this average to compensate for error caused by low resolution. The Amorphous Localization algorithm takes a different approach from the DV-Hop algorithm to estimate the average distance of a single hop. This task assumes that the density of the network, $\mathrm{n}_{\text {local }}$, is known a priori, so that it can calculate HopSize offline in accordance with the Kleinrock and Slivester formula[9].

$$
\text { hop size }=r\left(1+e^{-n_{\text {local }}}-\int_{-1}^{1} e^{-n_{\text {local }} \frac{\left(\cos t-t \sqrt{1-t^{2}}\right)}{\pi}} d t\right)
$$

Finally, after obtaining the estimated distances to three anchors, triangulation is used to estimate a node's location.

\subsubsection{Range free indoor localization system}

The active badge system is an indoor range-free system using infrared (IR) for signalling between the sensors and the badges worn by personnel. The location of a badge can be found given the positions of the sensors. A node localizes itself at the centroid of the overlapped transmission coverage regions of the beacon nodes. A set of connectivity constraints are built in, which are used to discover the location by convex optimization[5].

\section{Advanced Range Based Approaches}

The advanced range based approach is the one which is developed by using basic range based approach. Self localization and localization protocols are the two advanced range based approaches.

\subsection{Self Localization}

Unattended sensor networks are becoming increasingly important in a large number of military and civilian applications. The basic concept is to deploy a large number of low-cost, self-powered sensor nodes that acquire and process data. The sensor nodes may include one or more acoustic microphones as well as seismic, magnetic, or imaging sensors. A typical sensor network objective is to detect, track, and classify objects or events in the neighbourhood of the network.

\subsubsection{A New Methodology for localization.}

This method has two parts. The first part is concerned with the evaluation of the distance of the sensor nodes in accordance with the beacon nodes and the second part is concerned with finding the location of the sensor nodes in the sensor network. In this model, we control the transmission power of the beacon nodes such that we can conserve the energy of the beacon nodes. When the nodes are deployed these beacon nodes know their location and the other ordinary nodes find their location in accordance with the beacon nodes. Thus the beacon nodes play a very important role in the wireless sensor network. The main goal of this model is to conserve the power of the beacon node. This algorithm that locates the location of the sensor nodes in a wireless sensor network in a power efficient manner[13].

\subsubsection{Clustering Algorithm for localization}

A clustering algorithm which is designed to be used for localization purpose in wireless sensor networks. It is designed in such a way to consider principle of designing a clustering algorithm, in addition to providing an environment for designing a localization algorithm based on clustering. The proposed algorithm uses a weight function at each sensor node, which is a combination of different parameters including: reminding energy, transmission power and number of neighbours. Using the weight function the algorithm tries to classify the sensor nodes so that minimum number of clusters with maximum number of nodes in each cluster could be achieved. This algorithm selects a node that has enough energy for executing the task of head clustering. The numbers of transmitted messages in the algorithm are less than the other algorithms. Furthermore its execution time is acceptably lower than the other clustering algorithm which makes it suitable for localization purpose. The distribution of cluster on the network is so that the collection of cluster head can present a good image of the whole network [16]. 


\subsection{Localization Protocol}

Two approaches used in this protocols are range based approach and Minimum Enclosing Circle Containment approach.

\subsubsection{Range Based Centroid (RBC) approach}

It starts from a single beacon and is able to localize all the network nodes with an accuracy that improves on that of methods based on the sole measurement of range (that usually require multiple beacons). In order to contain the propagation of the localization error while the process progresses away from the beacon, we have also proposes a new localization protocol that achieves even greater accuracy[19].

\subsubsection{Minimum Enclosing Circle Containment $\left(\mathrm{MEC}^{2}\right)$ approach}

New localization protocol that achieves even greater accuracy termed $\mathrm{MEC}^{2}$ (for Minimum Enclosing Circle Containment). Observed that $\mathrm{MEC}^{2}$ keeps the localization error below $25 \%$ of the node transmission radius, with 20 to $30 \%$ improvements over RBC, and in general over common localization methods. We observe that combining range and angle of arrival produces better localization without requiring a higher number of beacons or extra hardware. The $\mathrm{MEC}^{2}$ solution, for instance, achieves accuracy which is acceptable for most geo routing and location aware applications with just one beacon[19].

\section{Advanced Range free Approaches}

Basic range free approaches used to develop the advanced range free approaches. Few advanced range free approaches discussed below.

\subsection{Dynamic Anchor distributed Localization}

A new distributed algorithm DA(dynamic anchor)is proposed to overcome the disadvantages of the DV-hop in the situation of nodes scattered uneven, quantities huge, and topology dynamically change. The DA promote an unknown node to be an anchor, and the area is divided into several parts, in each part, the DV-Hop is implemented, so the hops between the unknown node and the anchor decreased quickly which can overcome the disadvantages of the DV-hop algorithm. As the number of nodes increase, the hops between the unknown node and the anchor are increase too. To overcome this disadvantage of the DV-Hop, an unknown node is promoted to be an anchor, and the area is divided into several parts according to all the anchors, then the DVHop is implemented in each part. It is obvious that in each part, the hops between the unknown node and the anchor are decreased quickly, so more accurate coordinate is acquired by the DA [14]. Two estimated coordinates are acquired by the nodes in the public territory of two parts; the average coordinate is adopted to substitute the unknown node coordinate.

\subsection{Localization using Self Organizing Map}

\subsubsection{Self Organizing Map (SOM)}

A SOM is essentially a two-dimensional neural network that contains two layers of cells or nodes - an input layer and a network layer. SOM architecture is shown in figure 5. In the network layer, the nodes are arranged in a lattice-type array. This array can be constructed using any two- dimensional shape (e.g., a rectangular, square, or hexagon).A SOM projects the input space to the two-dimensional plane defined by the lattice of neurons. This property has been widely exploited in many applications for data analysis and visualization of large data sets. More recently, SOMs have been used to implement localization schemes [15]

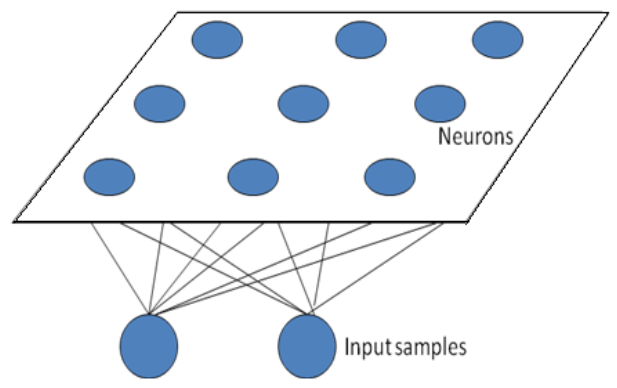

Fig.5. Architecture of SOM 


\subsubsection{Centralized localization based on SOM}

It works without anchor nodes and does not rely on range measurements. The algorithm is based on a specific neural network architecture known as a Self-organizing Map (SOM), which generates virtual coordinates that describe the relative positions of nodes. The approach is a centralized one, however, and is also limited to a small to medium size networks.

\subsubsection{Distributed localization Scheme}

The algorithm is based on the use of Self- Organizing Maps, which does not rely on any central nodes or anchor nodes. We demonstrate that by formulating a decentralized approach, the algorithm is efficient with computational burden in the order of $\mathrm{O}\left(\mathrm{N}^{2}\right)$; The algorithms can easily be implemented on a parallel or distributed parallel system.

\subsection{Mobile anchor Centroid localization}

Many wireless sensor network applications depend on nodes being able to accurately determine their locations. This is the first task to study range-free localization in the presence of mobility. The idea is that mobile anchor can improve the localization accuracy and coverage because it can move to every point of wireless sensor networks. Another one is that range-free has no extra hardware or data communication and reduces the costs of localization.

MACL technique can provide accurate localization even when memory limits are severe, the seed density is low, and network transmissions are highly irregular. MACL uses a single mobile anchor node as the reference node, which is required to move in the sensing field and broadcast its current position periodically. Sensor nodes receive the position information of the mobile node and localize themselves to the centroid of these positions by using the centroid algorithm [18].

\subsection{SSRML (Sector Scan RSSI Mobile Localization) Algorithm}

This localization algorithm presented for mobile wireless sensor networks and simulation experiments to evaluate the performance of the algorithms by varying the number of anchors, number of nodes and velocity of nodes.

The localization process of SSRML can be divided three steps, which are initializing system, predicting localization and filtering the prediction. After deployment, the unknown nodes estimate their localization first and predict the next one. After attaining the next prediction localization of the mobile nodes, the unknown nodes determine the current evaluation of the anchors' localization by using the receiving information from the anchors and the overlapping areas of the sector scan. After that, the localizations not existing in the overlapping areas on the previous prediction are filtered, then using the random nodes to replace the deleted prediction localization in this area. Do these steps again and again until the localization process to end [21].

The filtering approach indicates that the probability of each prediction localization is 0 or 1 . The prediction and filtering steps of the SSRML need to be initialized. This algorithm gives the localization accuracy can be minimized lower than $10 \%$

\subsection{Three -Dimension Localization based on SVM (Support Vector Machine)}

Support vector machine (SVM) is an excellent machine learning method, which is based on structural risk minimization principle and developed from statistical learning theory with strong generalization ability . SVM establishes the optimal classification surface in feature classes, therefore, has superior classification performance and has been applied in various fields. The experimental results demonstrate the algorithm proposed has small localization error and is insusceptible to the proportion of anchor nodes and range error. Hence, the algorithm proposed is robust and stable, which can be applied in network environment where anchor nodes are sparse and range error is large[20].

\section{Estimators for localization}

RSS measurements are usually inaccurate and error-prone, since they are affected by many factors such as multipath shadowing, interference and fading. Difference between the actual distance and calculated distance is called distance error. Distance error leads to inaccurate co-ordinates. Remedy for this is estimation of errors. Estimation concept provides accuracy.

\section{Conclusion}

Basic and Advanced Range based and Range free localization schemes discussed in this paper which will be useful for research. In future, we can make the localization process more secure so that the nodes can localize accurately even in the presence of attacker nodes. Some disadvantages are exist in the DA, it consumes more power than the DV-Hop. Building and controlling the real world applications of WSNs in self localization 
is by using 3-D models. Further research on three-dimension localization in WSN based on SVM is to be performed as future work, such as error analysis, quality and efficiency analysis of the approach, modified algorithm for optimization.

[1]. J. C. Navas and T. Imielinski, "Geographic Addressing and Routing," In Proc. Of the ACM MobiCom, Budapest Hungary, September 1997.

[2]. R. Burne, et. al, "A Self-Organizing, Cooperative UGS Network for Target Tracking," in the Proceedings of the SHE Conferenc Unattended Ground Sensor Technologies mid Applications II, Orlando Florida, April 2000.

[3]. N. Bulusu, J. Heidemann, and D. Estrin, "GPS-less Low-cost Outdoor Localization for Very Small Devices," ZEEE Personal Corizmruiicatiuns, 7(5):2\&34, October 2000

[4]. S. Capkun, M. Hamdi, and J.-P. Hubaux, "GPS-free Positioning in Mobile Adhoc Networks," in Hawaii International Conference on System Sciences (HICSS-34), pages 3481-3490, Maui, Hawaii, January 2001.

[5]. L. Doherty, K. Pister, and L. El Ghaoui, "Convex Position Estimation in Wireless Sensor Networks," in the Proceedings of lEEE Conference on Computer communication (iNFOCOM), Anchorage, Alaska, April 2001,

[6]. A. Sawides, C. C. Han, and M. Srivastava, "Dynamic Fine-Grained Localization in Ad-hoc Networks of Sensors," in the Proceedirigs of the rh IEEE/ACM Annual Conference on Mobile Computing networks(hfobiCUM'Ol), Rome, Italy, July 2001.

[7]. A. Savvides, H. Park, and M. B. Srivastava, "The Bits and Flops of The n-hop Multilateration Primitive for Node Localization Problems," in the Proceedings of [lie first ACM international workshop on Wireless Sensor Networks and Applications, Atlanta, GA, September 2002.

[8]. Y. Shang, et al., "Localization From Mere Connectivity," in the Proceedings of ACM MOBIHOC 2003, Annapolis, MD,June 2003.

[9]. Tian He,Chengdu Huang, Brian M. Blum "Range free localization Schemes for large scale sensor networks" MobiCom '03, September 14-19, 2003

[10]. X . Ji, "Sensor Positioning in Wireless Ad-hoc Sensor Networks with Multidimensional Scaling," in the Proceedings of IEEE INFOCOM, Hong Kong, March 2004.

[11]. X. Cheng, A. Thaeler, G. Xue, and D. Chen, "TPS: A Time- Based Positioning Scheme for Outdoor Sensor Networks," in the Proceedings of IEEE Conference on computer Communications (INFOCOM), Hong Kong, March 2004.

[12]. Mert Bal, Min Liu, Weiming Shen and Hamada Ghenniwa,"Localization in Cooperative Wireless Sensor Networks: A Review", Proceedings of the 2009 13th International Conference on Computer Supported Cooperative Work in Design

[13]. Allon Rai, Sangita Ale, and Syed S. Rizvi Aasia Riasat ," A New Methodology for Self Localization in Wireless Sensor Networks", Proceedings of the 12th IEEE International Multitopic Conference, December 23-24,2008

[14]. ZHAO Hu ,LI Zhi,"'Dynamic-Anchor Distributed Localization in Wireless Sensor Networks",IEEE 2008

[15]. Jie Hu and Gordon Lee," Distributed Localization of Wireless Sensor Networks Using Self-Organizing Maps",Proceedings of IEEE International Conference on Multisensor Fusion and Integration for Intelligent Systems Seoul, Korea, August 20 - 22, 2008.

[16]. Sadegh Zainalie,Mohammad Hossien Yaghmaee," CFL: A Clustering Algorithm For Localization in Wireless Sensor Networks", 2008 Internatioal Symposium on Telecommunications

[17]. Rajaa Alqudah and Stefano Basagni ," On the Effects of Multiple Beacons on Localization for Wireless Sensor Networks"IEEE 2008.

[18]. Zhen Hu1, Dongbing Gu2, Zhengxun Song1, Hongzuo Li,"Localization in Wireless Sensor Networks Using a Mobile Anchor Node" Proceedings of the 2008 IEEE/ASME International Conference on Advanced Intelligent Mechatronics July 2 - 5, 2008, Xi'an, China.

[19]. Xi-Rong Bao, Shi Zhang, Ding-Yu Xue,"Research on the Self-localization of Wireless Sensor Networks”, The 2008 International Conference on Embedded Software and Systems (ICESS2008).

[20]. Ruohong Ruan, Qingzhang Chen, Keji Mao, YunPan,” A Three-dimension Localization Algorithm for Wireless Sensor Network Nodes Based on SVM", IEEE 2010.

[21]. Tan Shun-hua, Chen Miao, Tang Tao," Localization Algorithm Based on Sector Scan for Mobile Wireless Sensor Networks", IEEE 2010.

[22]. P. Levis, D. Culler, “Mate: A Tiny Machine for Sensor Networks," Proceedings of (ASPLOS'02), San Jose, CA, Oct. 2002, pp. $85-95$. 